\title{
FPGA Based Wavelet Trigger in Radio Detection of Cosmic Rays
}

\author{
Zbigniew Szadkowski • Anna Szadkowska
}

Received: 6 February 2014 / Published online: 30 September 2014

(C) The Author(s) 2014. This article is published with open access at Springerlink.com

\begin{abstract}
Experiments which show coherent radio emission from extensive air showers induced by ultra-high-energy cosmic rays are designed for a detailed study of the development of the electromagnetic part of air showers. Radio detectors can operate with $100 \%$ up time as, e.g., surface detectors based on water-Cherenkov tanks. They are being developed for ground-based experiments (e.g., the Pierre Auger Observatory) as another type of air-shower detector in addition to fluorescence detectors, which operate with only $\sim 10 \%$ of duty on dark nights. The radio signals from air showers are caused by coherent emission from geomagnetic radiation and charge-excess processes. The self-triggers in radio detectors currently in use often generate a dense stream of data, which is analyzed afterwards. Huge amounts of registered data require significant manpower for off-line analysis. Improvement of trigger efficiency is a relevant factor. The wavelet trigger, which investigates on-line the power of radio signals $\left(\sim V^{2} / R\right)$, is promising; however, it requires some improvements with respect to current designs. In this work, Morlet wavelets with various scaling factors were used for an analysis of real data from the Auger Engineering Radio Array and for optimization of the utilization of the resources in an FPGA. The wavelet analysis showed that the power of events is concentrated mostly in a limited range of the frequency spectrum (consistent with a range imposed by the input ana$\log$ band-pass filter). However, we found several events with
\end{abstract}

Z. Szadkowski ( $\bowtie)$

Department of Physics and Applied Informatics, University of Łódź, Lodz, Poland

e-mail: zszadkow@uni.lodz.pl

\section{A. Szadkowska}

Center of Mathematics and Physics, Łódź,

University of Technology, Lodz, Poland suspicious spectral characteristics, where the signal power is spread over the full band-width sampled by a $200 \mathrm{MHz}$ digitizer with significant contribution of very high and very low frequencies. These events may not originate from cosmic ray showers but could be the result of human contamination. The engine of the wavelet analysis can be implemented in the modern powerful FPGAs and can remove suspicious events on-line to reduce the trigger rate.

Keywords FPGA $\cdot$ NIOS $®$ - Wavelet $\cdot$ Trigger $\cdot$ Radio detection $\cdot$ Geomagnetic radiation

\section{Introduction}

Several cosmic ray experiments (e.g., the Pierre Auger Observatory) located at ground level use a very large aperture detection system for the observation of ultra-highenergy cosmic rays (UHECRs). The radio technique is especially appropriate for the detection of UHECRs in large-scale arrays because of its almost $100 \%$ operational efficiency, its high angular resolution, and its sensitivity to longitudinal air-shower evolution.

The present challenges are to understand emission mechanisms and the features of the radio signal, and to develop an adequate measuring instrument. Electronpositron pairs generated in shower development are separated and deflected by the Earth's magnetic field, and hence introduce an electromagnetic emission [1], [2]. During shower development, charged particles are concentrated in a shower disk a few meters thick. This results in a coherent radio emission up to about $100 \mathrm{MHz}$. Short but coherent radio pulses from $10 \mathrm{~ns}$ to a few $100 \mathrm{~ns}$ are generated with an electric field strength increasing approximately linearly with the energy of the primary cosmic particle inducing 
the extended air showers (EAS). This results in quadratic dependence of the radio pulse energy as a function of the primary particle energy.

A current technique is estimation of the power in the radio band based on wavelet transformations. The main motivation for a development based on much more sophisticated algorithms is improvement of trigger efficiency. Most self-triggered registered events contain only noise. Significant improvement of trigger efficiency is a crucial factor. A lot of off-line data analyses require a nonnegligible amount of manpower. A much wiser approach would be the development of a much more efficient self-trigger. The presented wavelet trigger is an alternative proposal to the algorithms currently in operation.

\section{Wavelet Power Estimation}

Wavelet transform can be used to analyze time series which contain nonstationary power at many different frequencies [3]. At the Pierre Auger Observatory, the frequency window for the detection of cosmic rays by radio techniques has been placed in a "quiet" region $(\sim 30-80 \mathrm{MHz})$, i.e., below the FM and above strong transmitters operating at 20 to $25 \mathrm{MHz}[5,6]$.

Wavelet coefficients allow estimation of the power of a signal. The global wavelet power spectrum is defined as $\left|W_{k}(s)\right|^{2}[4]$ and the total power $\bar{W}^{2}(s)$ can be expressed as follows:

$\bar{W}^{2}(s)=\frac{1}{N} \sum_{k=0}^{N-1}\left|W_{k}(s)\right|^{2}=\frac{1}{N} \sum_{k=0}^{N-1}\left|\bar{X}_{k} \cdot \bar{\Psi}_{k}(s)\right|^{2}$

According to the Nyquist theorem, the sampling frequency in the ADCs digitizing the analog radio signals should be twice the value of the band width. The Auger Engineering Radio Array (AERA) selected 180 to $200 \mathrm{MHz}$ as the optimal sampling frequency. In order to cover the radio signal (with a typical length of $\sim 150 \mathrm{~ns}$ ) with the corresponding step size in a time of $5 \mathrm{~ns}$, the length of the corresponding transform (Eq. 1) should be at least 32.

Three families of reference Morlet wavelets are selected for the scaling factor $s^{-1}=\alpha=0.04,0.01$, and 0.004 , respectively:

$f_{\alpha, \text { freq }}(k)=\cos \left(2 \pi k \frac{\text { freq }}{\text { samplinglet }}\right) \exp \left(-\alpha \cdot k^{2}\right)$

where $-16 \leq k \leq 15$, sampling $=200(\mathrm{MHz})$ (Fig. 1).

Wavelets for $\alpha=0.04$ are squeezed to nonnegligible values in the range of $-10 \leq k \leq 10$. For $\alpha=0.004$, wavelets are stretched to a larger range beyond $-16 \leq k \leq$ 15. Figure 2 shows the FFT for all three scaling factors.

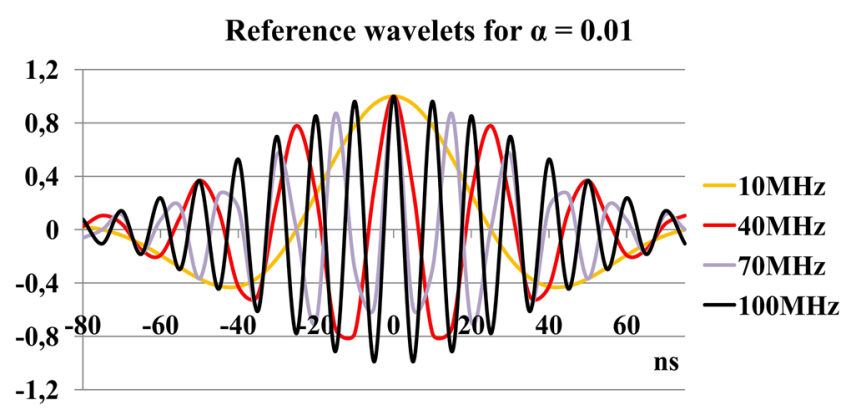

Fig. 1 The Morlet reference wavelets for $10-100 \mathrm{MHz}$ investigating range

$\left|\bar{X}_{k}\right|$ distributions are relatively wide for $\alpha=0.04$; separation between frequency ranges is limited. Much better separation is obtained for $\alpha=0.01$. Lower value $\alpha=0.004$ further reduces leakage between frequency bins. It has been selected for preliminary estimation of the signal power, although it corresponds to a longer stretch of time than the typical length of $150 \mathrm{~ns}$ and wavelets in the interval of $150 \mathrm{~ns}$ do not vanish completely as they do, for example, for $\alpha=0.01$ (Fig. 1).

The sum of the products of Fourier coefficients $\left(\bar{X}_{k}\right)$, calculated in the 32-point Fast Fourier Transform (FFT32) routine for ADC data $\left(x_{n}\right)$ (in each clock cycle), with precalculated Fourier coefficients of the reference wavelet $\left(\bar{\Psi}_{k}\right)$, gives an estimation of the power of selected types of wavelet (1). On-line power analysis requires simultaneous calculations of the power of many wavelets with the given scaling factor $s=\alpha^{-1}$.

The data acquisition system is equipped in the band-pass filter with lower and higher cutoff frequencies at 30 and $80 \mathrm{MHz}$, respectively. Figure $3 \mathrm{a}, \mathrm{b}$ show the energy distribution for a typical radio event. The energy is largely accumulated in a frequency range of $40 \leq$ freq $\leq 70 \mathrm{MHz}$. Figure $3 \mathrm{a}$ is calculated for the scaling factor $\alpha=0.04$ and presents a rough structure of the energy distribution.

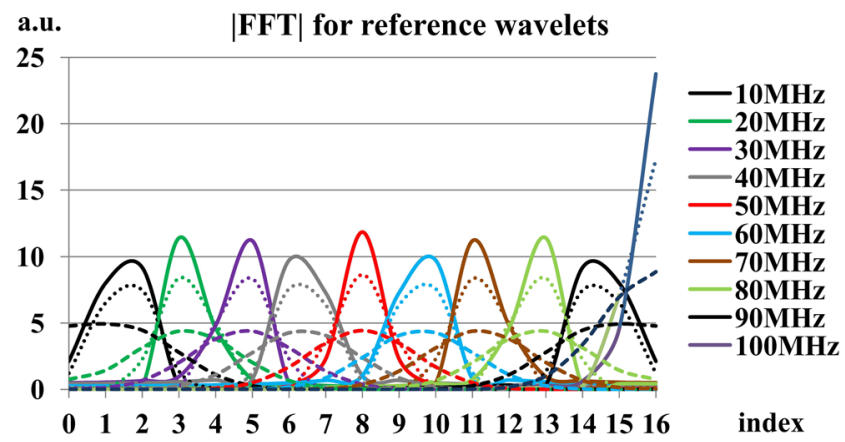

Fig. 2 The FFT spectra for reference wavelets for scaling factors : $\alpha=0.04$ (dashed), $\alpha=0.01$ (dotted), and $\alpha=0.004$ (continuous), respectively 

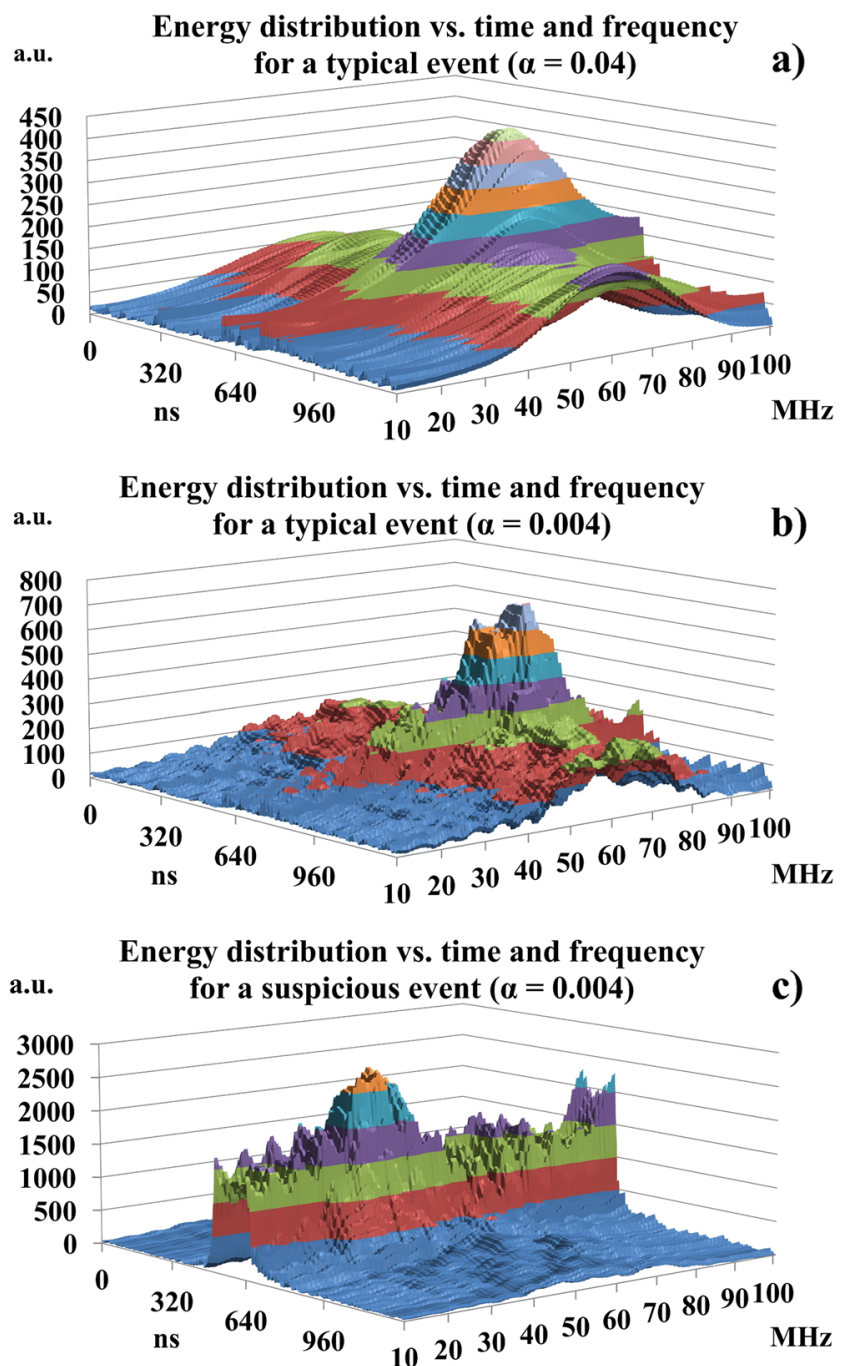

Fig. 3 An energy distribution as a function of time and frequency given for scaling factors $\alpha=0.04$ (upper graph) and $\alpha=0.004$ (middle graph)-typical events as well as an energy distribution for a suspicious event (lower graph $\alpha=0.004$ ). Colors are set automatically by the Excel package and denote only ranges of energy for arbitrarily selected grid. The same color assignment is applied for Figs. 4, 7, and 8

This is because of an overlap of the wavelet Fourier distributions and spectral leakage between neighboring frequency sectors. For the scaling factor $\alpha=0.004$, the energy distribution is reconstructed with very high precision (see Fig. 3b).

The analysis of AERA data allowed identification of several "suspicious" events with significantly different spectral characteristics (Fig. 3c) from "typical" ones (Fig. 3b). We can expect both destructive and constructive interferences between two different emission mechanisms, either of which could lead to apparently "strange" events. For example, Cherenkov beaming could result in very high signal strength in excess of $100 \mathrm{MHz}$. The energy for these events is not accumulated inside the relatively narrow frequency

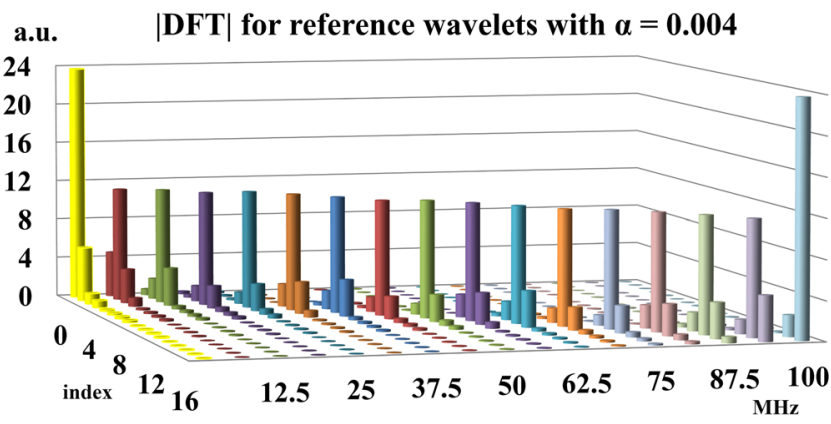

Fig. 4 The Fourier spectrum for 16 reference wavelets scaled for $\alpha=0.004$

band but spread over the full range of 10 to $100 \mathrm{MHz}$, with an additional significant contribution in the highest frequencies.

\section{FPGA Implementation}

The graphs in Fig. 3b, c are generated for 255 channels (255 reference wavelets). This number is certainly too large for implementation in the FPGA. The elementary component from Eq. (1) is a product of two complex numbers: Fourier coefficients $\left(\bar{X}_{k}\right)$ of real data $\left(x_{n}\right)$ and Fourier coefficients
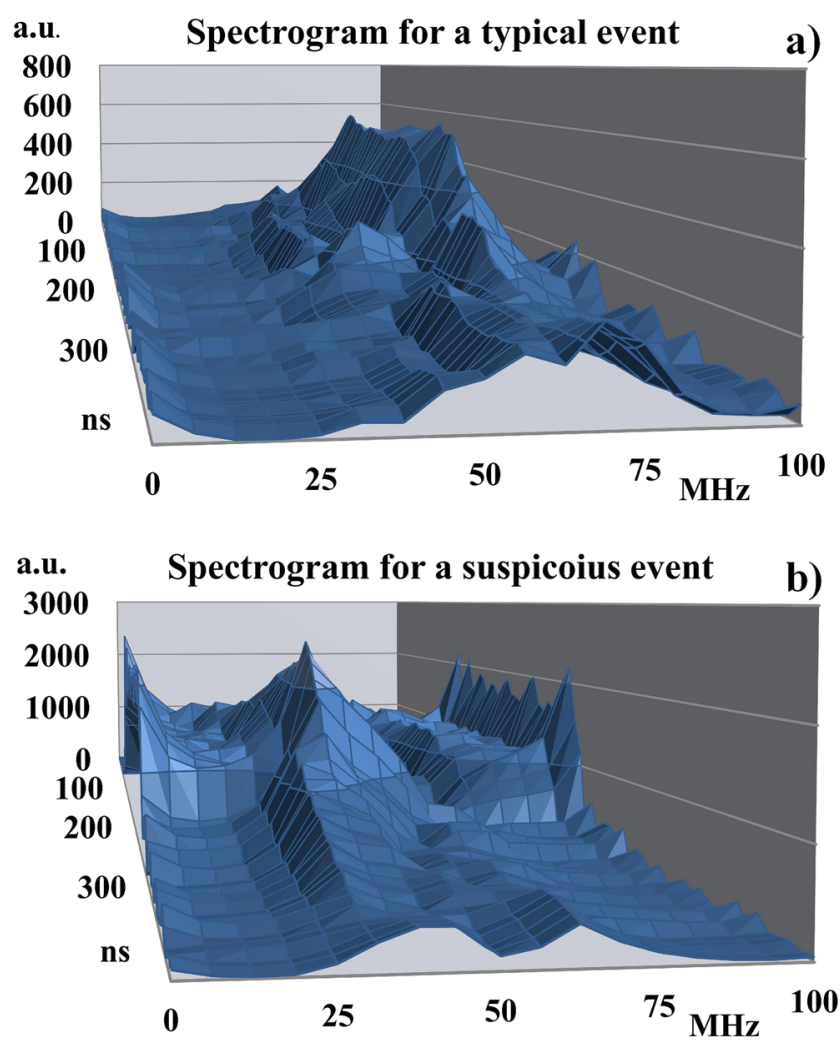

Fig. 5 Spectrograms for a typical and a suspicious events calculated for 16 wavelet engines with 2 indices for the power contribution 


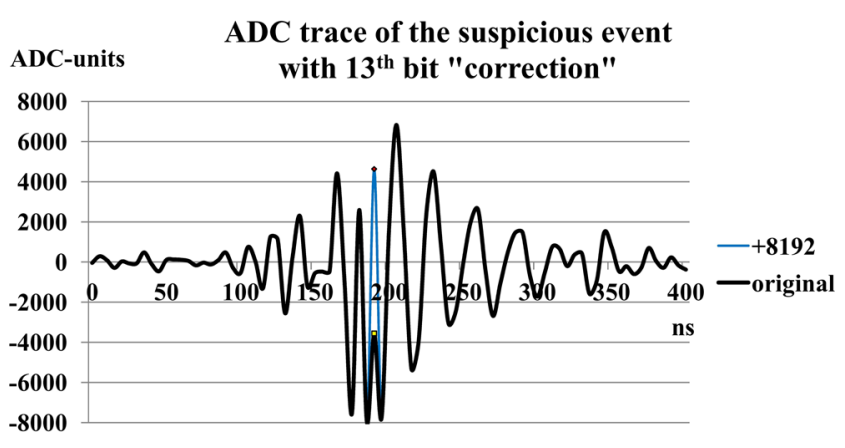

Fig. 6 The signal shape for a suspicious event with the spectrogram on Fig. 3c. The thin line corresponds to a "correction" of ADC value due to possible differential nonlinearity or delayed latch of a single bit

of the reference wavelet $\left(\bar{\Psi}_{k}\right)$. The simplest way to calculate this product on-line in the FPGA is to use the Altera ${ }^{\circledR}$ IP routine ALTMULT_COMPLEX. However, the wavelet spectral coefficients are predefined as constants for arbitrarily selected reference wavelets. The module of a complex product $\left|W_{k}\right|^{2}=\left|\bar{X}_{k} \cdot \bar{\Psi}_{k}\right|^{2}$ can be calculated as a real product of $\left|W_{k}\right|^{2}=\left|\bar{X}_{k}\right|^{2} \cdot\left|\bar{\Psi}_{k}\right|^{2}$.

The FFT32 routine utilizes 48 embedded (DSP) $18 \times 18$ multipliers. For a calculation of $15\left|W_{k}\right|^{2}\left(\left|W_{0}\right|^{2}\right.$ is trivial), the ALTMULT_ADD routine utilizes two DSP $18 \times 18$ multipliers for each index $\mathrm{k}, 30$ in total. $\left|W_{k}\right|^{2} \cdot\left|\Psi_{k}\right|^{2}$ products use the next 15 DSP $18 \times 18$ multipliers.

The biggest FPGA from the Cyclone ${ }^{\circledR} \mathrm{V}$ E family 5CEA9 (with logic only, and thus without an ARM-based hard processor system (HPS)) contains 684 DSP $18 \times$ 18 multipliers. This FPGA could implement FFT32 + 16 engines for various reference wavelets for two polarizations.

The biggest FPGA from the Cyclone ${ }^{\circledR}$ V SE family 5CSEA6 (with an ARM-based HPS) contains only 224 DSP $18 \times 18$ multipliers. These DSP multipliers are too few to implement a system with sufficient resolution (i.e., number of reference wavelets). It is crucial to reduce significantly the need for DSP block utilization, as FPGAs with an HPS are anticipated for the final design of the radio detectors.

Figure 4 shows the Fourier spectrum for only 16 reference wavelets, which are scaled for potentially large resolution ( $\alpha=0.004)$. The power contribution to the sum in Eq. (1) in fact comes from only two or three indices, independently of the frequency scale (Fig. 4). Thus, the wavelet engine can use far fewer DSP blocks by focusing on only those indices which make a significant contribution to the total power.

The FPGA utilizes 78 DSP blocks for a calculation of only $\left|W_{k}\right|^{2}(0 \leq \mathrm{k} \leq 16)$. According to the Fourier spectrum in Fig. 4, the partial power as a function of the frequency scale can be calculated for two indices with sufficient precision. For 16 reference wavelets, this requires $34=17 \times 2$ multipliers instead of $255=17 \times 15$ ones for a full analysis.

The implementation of this technique simultaneously in two independent, parallel channels (two polarizations) in the biggest FPGA from CycloneV from the SE, SX, or ST families (with HPS) is possible only if 112 DSP $18 \times 18$ multipliers can be utilized per channel. Seventyeight DSP blocks are required for calculation of only $\left|W_{k}\right|^{2}$. The rest (34 DSP blocks) is enough for an implementation of 16 reference wavelets, but with a contribution of only 2 indices.

\section{Analysis of Real Radio Data}

A set of just 16 reference wavelets allows reconstruction of the spectral characteristics of events and recognition of suspicious events with untypical spectrograms (compare Figs. $3 \mathrm{~b}, \mathrm{c}$ and $5 \mathrm{a}, \mathrm{b})$.

\subsection{Suspicious Events}

Figure 6 shows a trace which provides a spectrogram from Fig. 5b. One of the ADC values seems to be lower than is expected of signal integrity.

The "correction" (an artificial change of the most significant part to obtain an ADC shape more compatible with expectations-see the thin line in Fig. 6) does not help. Spectrograms in Fig. 7 show that such an artificial "correction" does not significantly change the energy contribution
Fig. 7 Spectrograms for a suspicious event with original and "corrected" ADC traces
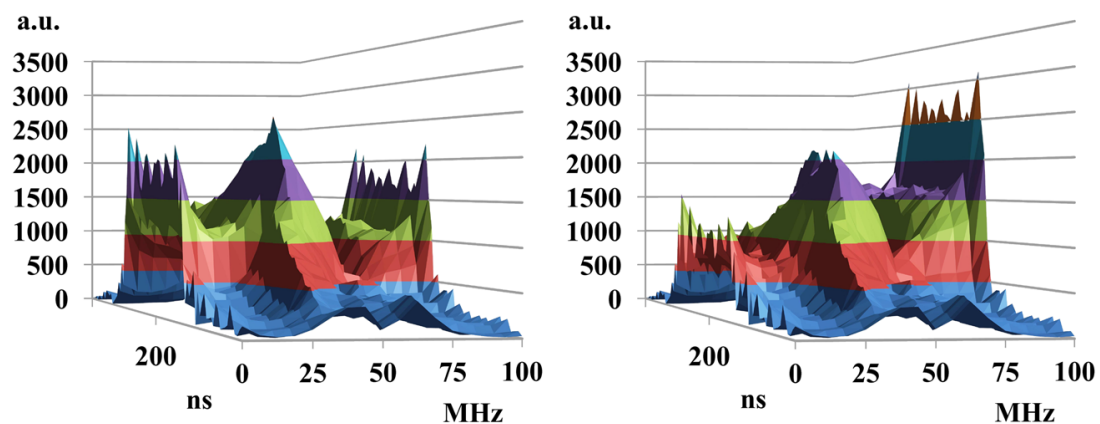
Fig. 8 Spectrograms for a typical event with original and artificially "distorted" ADC trace (an ADC value in a single time bin shifted on 1024 ADC-units)
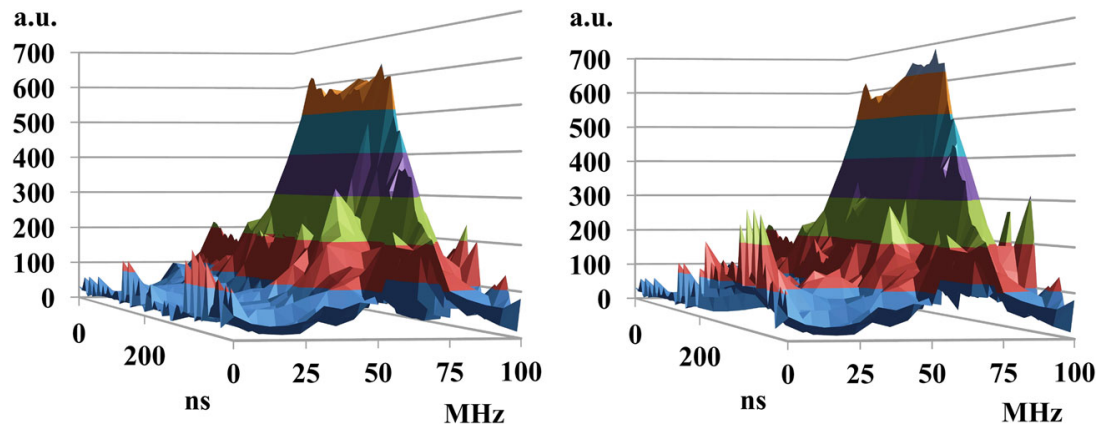

in the very high or very low-frequency ranges. This is to be expected because the change to the trace to make it look more like a "normal" trace is performed at only one time stamp. The change in the envelope (or integrated absolute pulse height) will thus be small.

This may suggest that the "suspicious" trace does not derive from the differential nonlinearity of the ADC or from a transmission failure from the ADC to the FPGA, but from some simultaneous process such as interference between geomagnetic and charge-excess processes.

Very high and very low frequencies should have been suppressed earlier by the input analog band-pass filter. However, strong human-made signals usually have a very wide frequency spectrum and spectral leakage through the input filters is a possibility.

\subsection{Typical Events}

Spectrograms are insensitive to artificial "correction" of the ADC trace in a single time bin (see Fig. 8). Spectral characteristics are immanent features of the event. Even a large artificial shift of the ADC value in a single time bin does not change the character of the spectrogram to any marked degree.

Thus, spectrograms can be used to classify events into different categories.

\subsection{Trigger}

The spectral energy contributions (for each reference wavelet) are integrated by the boxcar circuit [7], which averages the last 256 spectral samples (by means of memorybased shift registers). This reduces variation in the calculated signal power by a factor of 16 . Next, the spectral distortion factor is calculated as follows

$S D F=\sum_{k=0}^{4}\left|W_{k}\right|^{2}+\sum_{k=14}^{17}\left|W_{k}\right|^{2}-\sum_{k=5}^{14}\left|W_{k}\right|^{2}$

It selects events with a significant energy contribution in peripheral energy ranges. If the SDF 0 continuously for several (16) time bins, there is a high probability that the spectral characteristics of the current trace will be untypical (marked as "suspicious"). Averaging the power over 256 samples reduces the sensitivity of the SDF to fluctuations of power and reduces the probability of spurious classification. The analysis of these ADC traces confirms that their shapes have a similar character to the example in Fig. 6.

\section{Conclusions}

Spectrograms represent the global character of the registered event. They are weakly sensitive to sporadically appearing distortions like differential nonlinearity. Online spectral analysis may be used for recognition of suspicious events, whether highly contaminated or of human origin. To this end, a digitizer with a Cyclone ${ }^{\circledR} \mathrm{V}$ FPGA from the SX family (with HPS) is currently being developed.

The contribution of "suspicious" events in the analyzed AERA data (registered by the self-trigger) is less than $1 \%$. However, the data were registered by a particular algorithm of the trigger [8]. On-line wavelet analysis of unbiased data may support the existing trigger algorithm.

Wavelets have been used as a tool to support twodimensional analysis simultaneously in time and frequency domains to detect radio signals. The selection of a small scaling factor $\alpha$ reduces wavelet analysis to almost purely FFT on-line calculations. Nevertheless, it also allows identification of untypical (suspicious) events and reduction in the final size of the database through rejection of excessively noisy or significantly distorted signals.

Acknowledgments This work is being developed for the next generation of cosmic ray detectors supported by the ASPERA-2 consortium and was funded by the Polish National Center of Researches and Development under NCBiR Grant No. ERA /NET/ASPERA/02/11. Authors would like to thank Charles Timmermans from Radboud University Nijmegen, the Netherlands, for files with AERA radio events as well as many colleagues from the Pierre Auger Collaboration for valuable discussions, recommendation and hints. 
Open Access This article is distributed under the terms of the Creative Commons Attribution License which permits any use, distribution, and reproduction in any medium, provided the original author(s) and the source are credited.

\section{References}

1. H.R. Allan, Prog. in Elem. Part. and Cos. Ray Phys. 10, 171 (1971)

2. H. Falcke, P.W. Gorham, Astropart. Phys. 19, 477-494 (2003)
3. I. Daubechies, IEEE Trans. Inform. Theory. 36, 961-1004 (1990)

4. C. Torrence, G.P. Compo, Bull. Am. Meteorol. Soc. 79, 61-78 (1998)

5. Z. Szadkowski, E.D. Fraenkel, van den A.M. Berg, IEEE Real Time Conference (Berkeley, 2012)

6. A. Schmidt, H. Gemmeke, A. Haungs, K.-H. Kampert, C. Rühle, Z. Szadkowski, IEEE Trans. on Nucl. Science 58(4), 1621-1627 (2011)

7. H. Gemmeke, A. Grindler, H. Keim, M. Kleifges, N. Kunka, Z. Szadkowski, D. Tcherniakhovski, IEEE Trans. on Nucl. Science 47(2), 371-375 (2000). Part 1

8. J.L. Kelley, arXiv:pdf/1205.2104v1.pdf 